\title{
A SCHUR METHOD FOR THE SOLUTION OF THE MATRIX RICCATI EQUATION
}

\author{
MOHSEN RAZZAGHI \\ Department of Mathematics and Statistics, Mississippi State University \\ Mississippi State, MS 39762 and Department of Mathematics \\ Amirkabir University, Tehran, Iran.
}

(Received February 8, 1995)

\begin{abstract}
This paper is concerned with an analytic solution of the finite-time matrix Riccati equation. The solution to the Riccati equation is given in terms of multiple of two matrices. These matrices are found using a Schur-type decomposition for Hamiltonian matrices. Simple examples illustrating the method are presented.
\end{abstract}

KEYWORRDS AND PHRASES. Schur method, matrix Riccati, analytic solution. 1992 AMS SUBJECT CLASSIFICATION CODES. 49B10, $93 \mathrm{~B} 40$

\section{INTRODUCTION.}

The matrix Riccati equation appears in most optimal control systems design problems. This equation, in one form or another, has an important role in linear state and output regulator tracking, multi-variable and large scale systems, scattering theory and estimation $[1,2]$.

It is known that the boundedness of the solution of the matrix Riccati equation is equivalent to the "no-conjugate point to the final time" [3-5]. The solution of this equation is difficult to obtain from two points of view. One is that it is nonlinear, and the other being in matrix form. In the present paper the solution to the matrix Riccati equation is expressed in terms of multiple of two matrices. These matrices are then obtained by using a Schur-type decomposition for Hamiltonian matrices. Illustrative examples are presented to show the feasibility of this method.

\section{STATEMENT OF THE PROBLEM}

Consider the linear time invariant system,

$$
\dot{x}(t)=A x(t)+B u(t), x\left(t_{0}\right)=x_{0}
$$

where $x(t)$ and $u(t)$ are $n \times 1$ and $m \times 1$ state and control vectors, respectively. A and B are constant $n \times n$ and $n \times m$ matrices. The optimal control problem would be to find an optimal control $u(t)$ satisfying (2.1) while minimizing the quadratic cost functional,

$$
J=\frac{1}{2} x^{T}\left(t_{f}\right) S x\left(t_{f}\right)+\frac{1}{2} \int_{t_{0}}^{t_{f}}\left[x^{T}(t) Q x(t)+u^{T}(t) R u(t)\right] d t
$$

where $t_{0}$ and $t_{f}$ are the initial and final times which are given, $S, Q$, and $R$ are constant matrices of appropriate dimensions, $R$ is symmetric positive definite, while $S$ and $Q$ are symmetric positive semidefinite, and $T$ denotes transpositions. The optimal control is given by [6]

$$
u(t)=-R^{-1} B^{T} W(t) x(t)
$$


where $W(t)$ is the solution of the matrix Riccati equation

$$
\dot{W}=-W D_{1}-D_{1}^{T} W-W D_{2} W+D_{3}, W\left(t_{f}\right)=S
$$

with

$$
D_{1}=A, D_{2}=-B R^{-1} B^{T}, D_{3}=-Q .
$$

Usually, the infinite time problem is considered, in this case $\dot{W}=0$ and the Riccati equation is reduced to an algebraic equation. The solution of the matrix Riccati equation has been considered in [6-8]. The solution of (2.3) can be expressed as [8].

$$
W(t)=Y(t) X^{-1}(t) \text { with } Y\left(t_{f}\right)=S, X\left(t_{f}\right)=I
$$

where

$$
\dot{Z}=D Z
$$

with

$$
Z(t)=\left[\begin{array}{c}
X(t) \\
Y(t)
\end{array}\right] \text { and } D=\left[\begin{array}{cc}
D_{1} & D_{2} \\
D_{3} & -D_{1}^{T}
\end{array}\right]
$$

The solution of (2.5) is given by

$$
Z(t)=e^{D\left(t-t_{f}\right)} \quad\left[\begin{array}{l}
X\left(t_{f}\right) \\
Y\left(t_{f}\right)
\end{array}\right]
$$

Let $t-t_{f}=t_{1}$. Then (2.7) can be written as

$$
Z(t)=M \exp \left(M^{-1} D M t_{1}\right) M^{-1}\left[\begin{array}{l}
I \\
S
\end{array}\right]
$$

where $M$ is a $2 n \times 2 n$ nonsingular matrix. It is known that [9]

$$
e^{\left[\begin{array}{ll}
A & B \\
O & C
\end{array}\right] t}=\left[\begin{array}{cc}
e^{A t} & \int_{0}^{t} e^{A(t-s)} B e^{C s} d s \\
0 & e^{C t}
\end{array}\right]
$$

where $A, B$ and $C$ are square matrices. To find $Z(t)$ in (2.8) we find $M$ which makes $M^{-1} D M$ a quasiupper-triangular. This can be achieved using a Schur method and leads to a method for obtaining the solution to the Riccati equation.

\section{SOLUTION OF THE MATRIX RICCATI EQUATION USING A SCHUR METHOD}

We will use the following theorems.

THEOREM 1. (Schur canonical form). There exists a unitary matrix $U$ such that $U^{H} G U$ is triangular and has as diagonal elements the eigenvalues of $G$. Also there exists an orthogonal matrix $P$ such that $P^{T} G P$ is quasi- upper-triangular where each diagonal element is either a $1 \times 1$ matrix or a $2 \times 2$ matrix having complex conjugate eigenvalues [10].

THEOREM 2. (The real Schur-Hamilton decomposition). If $D$ has no pure imaginary eigenvalues, then there exist an orthogonal matrix $Q$

$$
Q=\left[\begin{array}{rr}
Q_{11} & Q_{12} \\
-Q_{12} & Q_{11}
\end{array}\right] \quad Q_{11}, Q_{12} \epsilon R^{n \times n}
$$

such that

$$
G=Q^{T} D Q=\left[\begin{array}{rr}
E & F \\
0 & -E^{T}
\end{array}\right] \quad E, F \epsilon R^{n \times n}
$$

where $E$ is upper quasitriangular with $F^{T}=F$. Also, $Q$ can be chosen such that the eigenvalues of $E$ are in the left half plane and moreover, each $2 \times 2$ diagonal block of $E$ appears with a complex conjugate pair of eigenvalues [11]. 
If $D$ has no pure imaginary eigenvalues, then we choose $M$ in (2.8) using Theorem 2 . Hence, we have

$$
\left[\begin{array}{l}
X \\
Y
\end{array}\right]=\left[\begin{array}{rr}
Q_{11} & Q_{12} \\
-Q_{12} & Q_{11}
\end{array}\right]\left[\begin{array}{cc}
e^{E t_{1}} & \int_{0}^{t_{1}} e^{E\left(t_{1}-s\right)} F e^{-E^{T} s} d s \\
0 & e^{-E t_{1}}
\end{array}\right]\left[\begin{array}{rr}
Q_{11} & Q_{12} \\
-Q_{12} & Q_{11}
\end{array}\right]^{T}\left[\begin{array}{l}
I \\
S
\end{array}\right]
$$

and if $D$ has pure imaginary eigenvalues, we use Theorem 1 . Consequently, matrices $X$ and $Y$ can be calculated. The results obtained are illustrated by the following examples.

\section{EXAMPLES}

Example 1. Consider minimizing the cost functional

$$
J=\frac{1}{2} \int_{0}^{t_{f}}\left[x^{T}(t)\left[\begin{array}{ll}
1 & 0 \\
0 & 2
\end{array}\right] x(t)+u^{T}(t) u(t)\right] d t
$$

subject to the conditions

$$
\dot{x}(t)=\left[\begin{array}{ll}
0 & 1 \\
0 & 0
\end{array}\right] x(t)+\left[\begin{array}{l}
0 \\
1
\end{array}\right] u(t), x(0)=x_{0} .
$$

In this problem using (2.6), we obtain

$$
D=\left[\begin{array}{rrrr}
0 & 1 & 0 & 0 \\
0 & 0 & 0 & -1 \\
-1 & 0 & 0 & 0 \\
0 & -2 & -1 & 0
\end{array}\right]
$$

The eigenvalues of $D$ are $-1,-1,1$ and 1 . The matrix $Q$,

$$
Q=\left[\begin{array}{rrrr}
\frac{1}{2} & \frac{\sqrt{5}}{10} & -\frac{1}{2} & -\frac{3 \sqrt{5}}{10} \\
-\frac{1}{2} & \frac{\sqrt{5}}{10} & \frac{1}{2} & -\frac{3 \sqrt{5}}{10} \\
\frac{1}{2} & \frac{3 \sqrt{5}}{10} & \frac{1}{2} & \frac{\sqrt{5}}{10} \\
-\frac{1}{2} & \frac{3 \sqrt{5}}{10} & -\frac{1}{2} & \frac{\sqrt{5}}{10}
\end{array}\right]
$$

in an orthogonal matrix which redures $D$ to

$$
G=Q^{T} D Q=\left[\begin{array}{rr}
E & F \\
0 & -E^{T}
\end{array}\right]
$$

with

$$
E=\left[\begin{array}{rr}
-1 & \frac{2 \sqrt{5}}{5} \\
0 & -1
\end{array}\right] \text { and } F=\left[\begin{array}{cc}
1 & -\frac{\sqrt{5}}{5} \\
-\frac{\sqrt{5}}{5} & 1
\end{array}\right]
$$

also, $e^{E t_{1}}$ and $e^{-E^{T} t_{1}}$ can be calculated as

$$
e^{E t_{1}}=\left[\begin{array}{cc}
e^{-t_{1}} & \frac{2 \sqrt{5}}{5} t_{1} e^{-t_{1}} \\
0 & e^{-t_{1}}
\end{array}\right] \text { and } e^{-E^{T} t_{1}}=\left[\begin{array}{cc}
e^{t_{1}} & 0 \\
-\frac{2 \sqrt{5}}{5} t_{1} e^{t_{1}} & e^{t_{1}}
\end{array}\right]
$$

Hence, using (3.3) with $S=\left[\begin{array}{ll}0 & 0 \\ 0 & 0\end{array}\right]$, we obtain

$$
\begin{gathered}
X=\frac{1}{2}\left[\begin{array}{cc}
t_{1} \cosh t_{1}-t_{1} \sinh t_{1} & \sinh t_{1}+t_{1} \cosh t_{1} \\
\sinh t_{1}-t_{1} \cosh t_{1} & 2 \cosh t_{1}+t_{1} \sinh t_{1}
\end{array}\right] \\
Y=\frac{1}{2}\left[\begin{array}{cc}
t_{1} \cosh t_{1}-3 \sinh t_{1} & -t_{1} \sinh t_{1} \\
t_{1} \sinh t_{1} & -\left(3 \sinh t_{1}+t_{1} \cosh t_{1}\right)
\end{array}\right] .
\end{gathered}
$$

Then

$$
\begin{aligned}
& W=Y X^{-1} \\
& \quad \frac{1}{3 \cosh 2 t_{1}+2 t_{1}^{2}+5}\left[\begin{array}{cc}
-6 \sinh 2 t_{1}+4 t_{1} & 3 \cosh 2 t_{1}-2 t_{1}^{2}-3 \\
3 \cosh 2 t_{1}-2 t_{1}^{2}-3 & -6 \sinh 2 t_{1}+4 t_{1}
\end{array}\right]
\end{aligned}
$$

Example 2. The following example was first given in [9]. 
Find the solution of the following Riccati equation:

$$
\dot{P}=P^{2}+2 P+2, P\left(t_{f}\right)=1 \text {. }
$$

For this problem, the matrix $D$ is given by

$$
D=\left[\begin{array}{rr}
-1 & -1 \\
2 & 1
\end{array}\right]
$$

and the matrix

$$
U=\frac{1}{\sqrt{6}}\left[\begin{array}{cc}
i-1 & 2 \\
2 & i+1
\end{array}\right]
$$

is a unitary matrix which reduces $D$ to

$$
U^{H} D U=\left[\begin{array}{cc}
i & i+2 \\
0 & -i
\end{array}\right]
$$

Then

$$
\left[\begin{array}{l}
X \\
Y
\end{array}\right]=\frac{1}{6}\left[\begin{array}{cc}
i-1 & 2 \\
2 & i+1
\end{array}\right]\left[\begin{array}{cc}
e^{i t_{1}} & (i+2) \sin t_{1} \\
0 & e^{-i t_{1}}
\end{array}\right]\left[\begin{array}{cc}
-i-1 & 2 \\
2 & -i+1
\end{array}\right]\left[\begin{array}{l}
1 \\
1
\end{array}\right]
$$

and

$$
P(t)=\frac{Y}{X}=\frac{\cos t_{1}+3 \sin t_{1}}{\cos t_{1}-2 \sin t_{1}} .
$$

This is in agreement with the result obtained in [9].

\section{CONCLUSION}

A method is proposed for the solution of the matrix Riccati equation which has several applications in optimal control problems. This method transforms the problem into examining the exponential of the Hamiltonian matrix. This exponential is found using a Schur decomposition for Hamiltonian matrices. Examples illustrating the concept involved are included.

\section{REFERENCES}

1. JAMSHIDI, M., An overview on the solutions of the algebraic matrix Riccati equation and related problems, Large Scale Systems, 1, (1980), 167-192.

2. REID, W.T., Riccati Differential Equations, Academic Press (1972), New York.

3. BREAKWELL, J.V. and HO, Y.C., On the conjugate point condition for the control problem, Int. J. Eng. Sci., 2, (1965), 567-579.

4. RAZZAGHI, M. and JAMSHIDI, M., On the Jacobi conditions for control problems with input delay, Proc. IEE., 122, (1975), 1313-1315.

5. RAZZAGHI, M., Conjugate point conditions for linear quadratic control problems Aut. Contr. Theory and Appl., 6, No. 3, (1978), 51-53.

6. RAZZAGHI, M., Solution of the matrix Riccati equation in optimal control Information Sci., 16, (1978), 61-73.

7. JODAR, L. AND NAVARRO, E., Closed analytical solution of Riccati type matrix differential equations Indian J. Pure and Appl. Math, 23, (1992), 185-187.

8. RAZZAGHI, M., A computational solution for a matrix Riccati differential equation Numerische Math., 32, (1979), 271-279.

9. SASAGAWA, T., A necessary and sufficient condition for the solution of the Riccati equation to be periodic IEEE Trans. Automat. Contr., AC-25, (1980), 564-566.

10. GOLUB, G.H. AND VAN LOAN, C.F., Matrix computations, The John's Hopkins University Press, (1983)

11. PAIGE, C. AND VAN LOAN, C., A Schur decomposition for Hamiltonian matrices Linear Algeb. and Its Appl., 41, (1981), 11-32. 


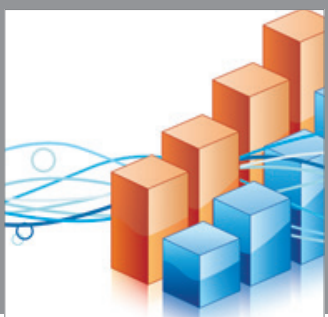

Advances in

Operations Research

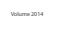

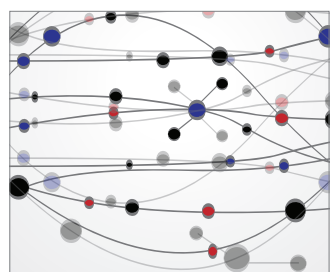

\section{The Scientific} World Journal
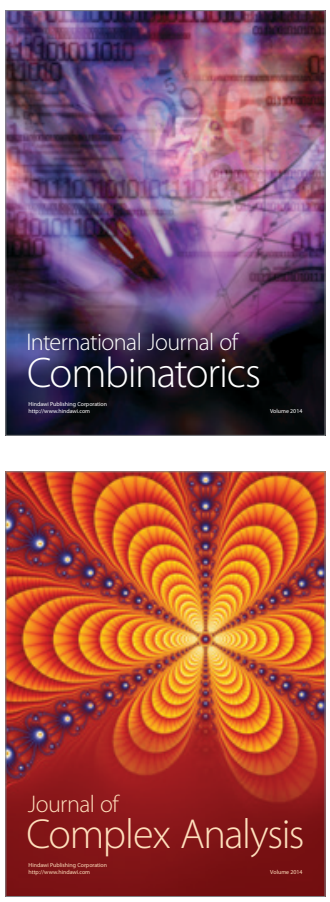

International Journal of

Mathematics and

Mathematical

Sciences
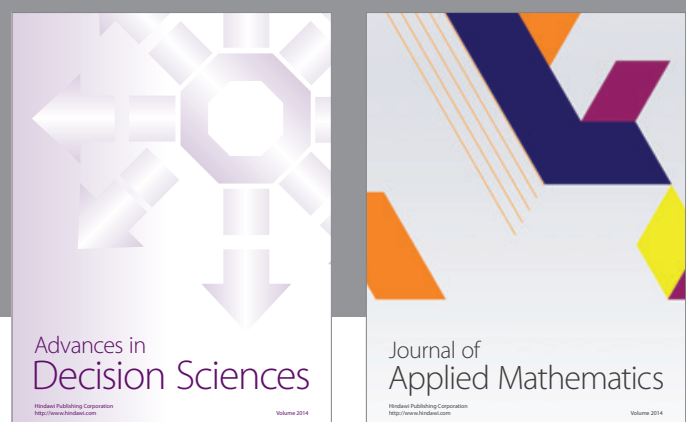

Journal of

Applied Mathematics
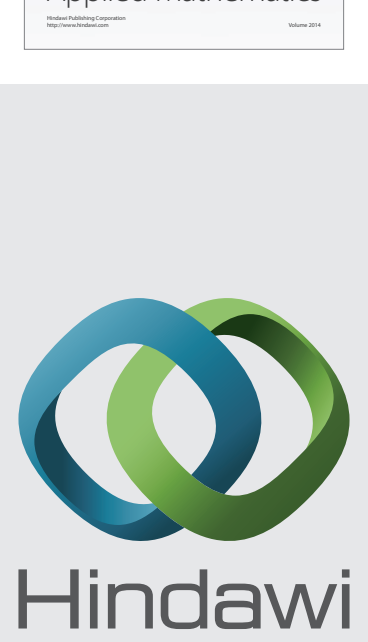

Submit your manuscripts at http://www.hindawi.com
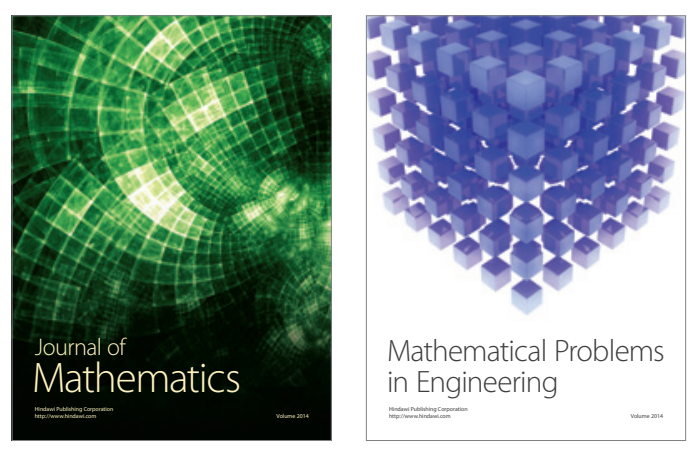

Mathematical Problems in Engineering
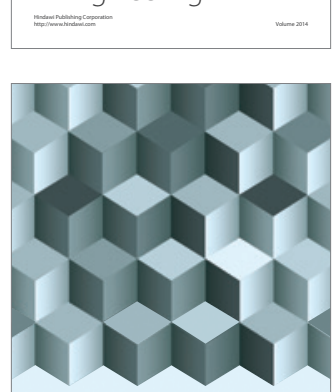

Journal of

Function Spaces
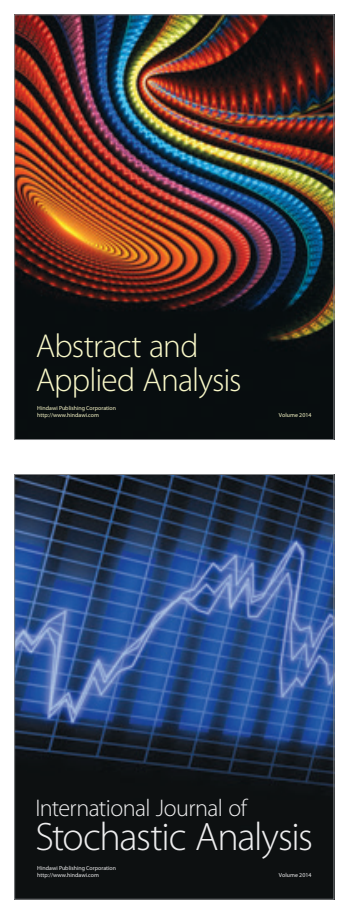

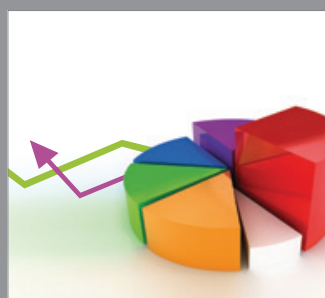

ournal of

Probability and Statistics

Promensencen
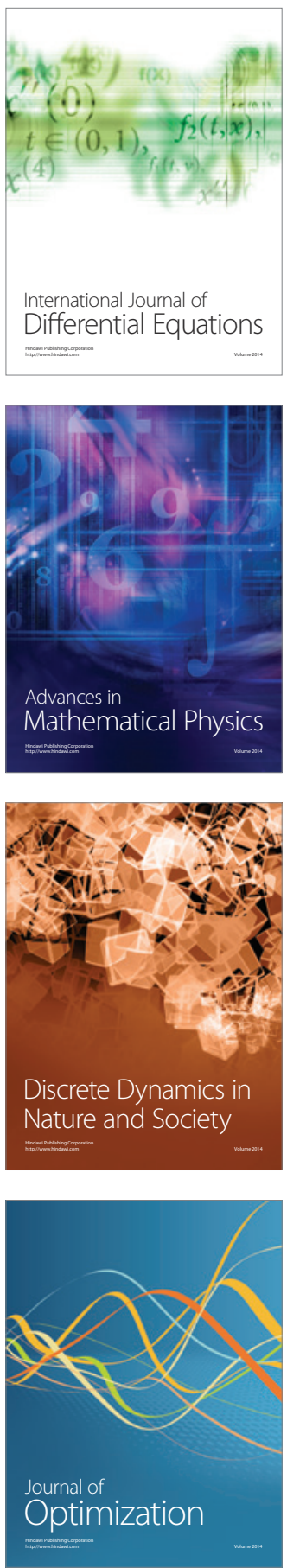